\title{
Experimental analysis and computational modeling of interburst intervals in spontaneous activity of cortical neuronal culture
}

\author{
T. Gritsun · J. le Feber · J. Stegenga · W. L. C. Rutten
}

Received: 19 July 2010 / Accepted: 16 June 2011 / Published online: 27 October 2011

(C) The Author(s) 2011. This article is published with open access at Springerlink.com

\begin{abstract}
Rhythmic bursting is the most striking behavior of cultured cortical networks and may start in the second week after plating. In this study, we focus on the intervals between spontaneously occurring bursts, and compare experimentally recorded values with model simulations. In the models, we use standard neurons and synapses, with physiologically plausible parameters taken from literature. All networks had a random recurrent architecture with sparsely connected neurons. The number of neurons varied between 500 and 5,000. We find that network models with homogeneous synaptic strengths produce asynchronous spiking or stable regular bursts. The latter, however, are in a range not seen in recordings. By increasing the synaptic strength in a (randomly chosen) subset of neurons, our simulations show interburst intervals (IBIs) that agree better with in vitro experiments. In this regime, called weakly synchronized, the models produce irregular network bursts, which are initiated by neurons with relatively stronger synapses. In some noise-driven networks, a subthreshold, deterministic, input is applied to neurons with strong synapses, to mimic pacemaker network drive. We show that models with such "intrinsically active neurons" (pacemaker-driven models) tend to generate IBIs that are determined by the frequency of the fastest pacemaker and do not resemble experimental data. Alternatively, noise-driven models yield realistic IBIs. Generally, we found that large-scale noise-driven neuronal network models required synaptic strengths with a bimodal distribution to reproduce the experimentally observed IBI range. Our results imply that the results obtained from small network models cannot simply be extrapolated to models of more realistic
\end{abstract}

T. Gritsun · J. le Feber · J. Stegenga · W. L. C. Rutten $(\bowtie)$

Neural Engineering Department, Institute for Biomedical Engineering MIRA, University of Twente, Enschede, The Netherlands

e-mail: t.gritsun@utwente.nl size. Synaptic strengths in large-scale neuronal network simulations need readjustment to a bimodal distribution, whereas small networks do not require such changes.

Keywords Cultured neuronal networks · Interburst interval - Random recurrent neuronal network models . Bimodal networks · Noise- and pacemaker-driven network simulations

\section{Introduction}

Biologically inspired artificial neural networks have major applications in artificial intelligence and signal processing. However, none of them can achieve the processing power of the biological original. To bring artificial networks closer to nature, we need a better understanding of biological neural networks. Neuronal cultures provide a good basis for that.

The most striking property of spontaneously firing cultures is their regular bursting activity, a burst being defined as more or less synchronized firing in sets of neurons spread throughout the whole network. The regularity of bursting may change gradually with time, typically being stable over hours (Stegenga et al. 2008). Cultured cortical networks composed of many thousands of neurons show bursting behavior starting from the end of the first week in vitro. Bursts can be characterized by both intraburst parameters (burst shape, maximum firing rate, leading and trailing edge steepness, etc.) and interburst parameters (statistics, stability of burst rates) (Van Pelt et al. 2004; Wagenaar et al. 2006). Cultured cortical networks usually show bursting activity at several different time scales; for instance, Baker et al. (2006) measured such bursting behavior in organotypic mega-cocultures of neonatal rat cerebral cortex, and distinguished so-called network bursts, minibursts, and microbursts, based 
on spike clustering analysis. It is necessary to stress that burst profiles usually have highly variable features in their leading and trailing slopes (Gritsun et al. 2010), and can be easily entangled with subsequent bursts. The latter so-called superbursts are not uncommon in cortical neuronal cultures (Wagenaar et al. 2006) and may last up to several seconds.

In a previous study (Gritsun et al. 2010) we modeled the intraburst phenomena, whereas in this study we focus on the interburst intervals (IBIs). As before, we will compare models with experimental outcomes.

Only a few studies on cultured cortical networks provide rough data on ranges and regularity of IBIs, and the data are mostly acquired from rather short epochs of recordings. The most extensive study (Wagenaar et al. 2006) observed clear IBI spans ranging from about 0.1 to $900 \mathrm{~s}$ in 30- min samples. It was noticed that IBIs in individual samples were narrowly distributed in most recordings, in contrast to Segev et al. (2002), who suggested that experimental data follow scale-free (Levy) distributions similar to those of interspike intervals (ISI) (Segev et al. 2001). In modeling studies by Giugliano et al. (2004) it was shown that IBIs are irregular and follow a Poisson point process with a dead time, while ISIs are usually much more irregular. They also suggested that cortical networks tend to keep that irregularity of bursting over the entire span of their active lifetime while neuronal development and synaptic plasticity constantly alter intrinsic network features. Consequently, in this study we intend to clarify the degree of regularity of the data and to find out which distribution best fits the experimental IBIs.

Much effort has recently been made to explain burst interval dynamics using several network models, e.g., Tsodyks et al. (1998), Tsodyks et al. (2000), and Wiedemann and Luthi (2003). Most of these models have networks with up to 1,000 neurons and with stationary ranges of input parameters (Amit and Brunel, 1997; Segev et al. 2001; Giugliano et al. 2004; French and Gruenstein 2006). Amit and Brunel (1997) suggested that, in case of a constant number of connections per neuron, a homogeneous network would switch from synchronous to asynchronous activity by increasing the number of neurons (to infinity), while others, using analytical approximations, indicated that activity dynamics would not change with monotonously (homogeneously) readjusted synaptic weights, e.g., Hansel and Sompolinsky (1996). However, network size is seen to have a large impact on bursting patterns in experimental data (Wagenaar et al. 2006).

In previous work we successfully investigated network models of 5,000 neurons with heterogeneously distributed synaptic weights (Gritsun et al. 2010). In this study we proceed with models incorporating 500-5,000 neurons. They are simulated for 1-h epochs. This enables us to examine how network size and connection probability influence synchronization of spiking activity. We apply broader ranges of connection probabilities, synaptic noise, and other parameters that can dramatically change during culture development. All simulated networks have wide (but physiologically plausible) ranges of input parameters, thus enabling the models to work in several operational regimes; for example, while changing excitatory synaptic strengths, Giugliano et al. (2004) described three qualitatively different regimes including asynchronous, synchronous, and high-rate asynchronous. Here we report on several model regimes. However, only one could reproduce the experimental ranges of IBIs while also keeping intraburst parameters within physiologically plausible ranges. In particular, we focused on the relationship between the distribution of synaptic strengths (homogeneous, bimodal) and the resulting operating regimes. The latter were expected to differ a lot for large and small networks.

\section{Methods}

\subsection{Cultures and recordings}

In our work we use culturing, recording, and other experimental techniques explained in detail by Stegenga et al. (2008). In brief, cortical neurons were obtained from either newborn or E18 Wistar rats. The dissociated cells were plated at concentration of $10^{6}$ cells $/ \mathrm{ml}$ and were allowed to adhere for $2 \mathrm{~h}$. Multi-electrode arrays (MEA) were coated in advance with polyethyleneimine to increase adhesion. Then, the nonadhering cells were removed by refreshing the medium, and $600 \mu 1$ R12 medium was added (Romijn et al. 1984). The resulting monolayer had a density of about 5,000 cells $/ \mathrm{mm}^{2}$. Two-thirds of the medium was changed twice a week for all cultures; all other conditions were kept constant. The cultures were stored in an incubator at $37^{\circ} \mathrm{C}$ at $\mathrm{CO}_{2}$ concentration of $5 \%$ and near $100 \%$ humidity. Figure 1 shows a part of a cultured neuronal network 10 days after plating. Usually, cultures were almost completely free of aggregation.

We used a MC1060BC setup and MEAs from MultiChannel Systems GmbH. The MEAs had 60 titanium-nitride electrodes in an $8 \times 8$ square grid. The interelectrode distance was $100 \mu \mathrm{m}$, and the diameter of the electrodes was $10 \mu \mathrm{m}$. The temperature was controlled at $36^{\circ} \mathrm{C}$, and a $\mathrm{CO}_{2}$ concentration of $5 \%$ was maintained. Custom-made LabView (National Instruments, Austin, TX) programs were made to control data acquisition.

\subsection{Simulation model}

We used the same network model with a random recurrent scale-free connection topology as in a previous study that focused on intraburst characteristics (Gritsun et al. 2010). It is described by the following set of equations: 


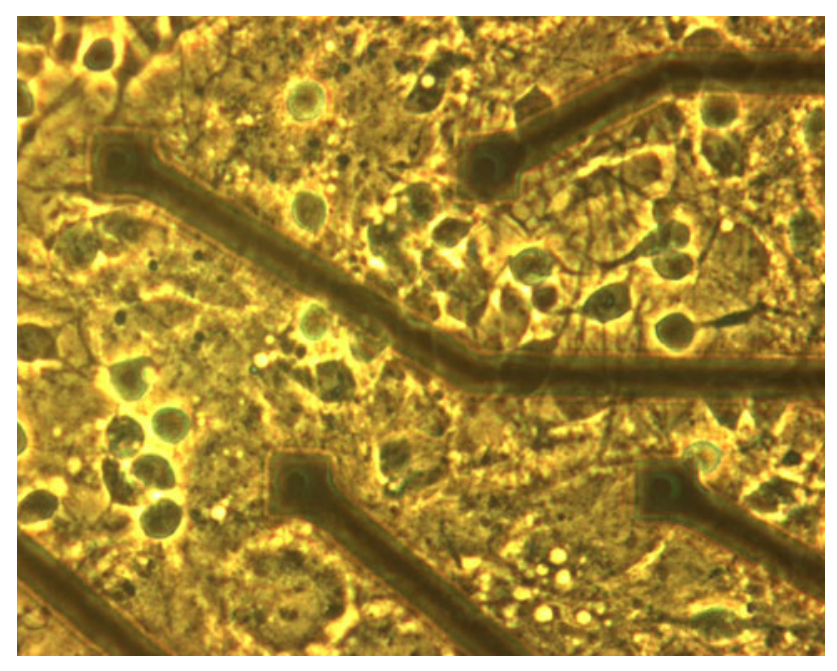

Fig. 1 Microphotograph showing part of a cultured neuronal network surrounding 4 electrodes (out of 60), 10 days after plating. Electrode diameter is $10 \mu \mathrm{m}$

$x_{i}(t+\mathrm{d} t)=\left\{\begin{array}{ll}1, & \text { if } f_{i}\left(v_{i}, w_{i j}, x_{j}\right) \geq 30 \mathrm{mV} \\ 0, & \text { otherwise }\end{array}\right.$,

where

$f_{i}\left(v_{i}, w_{i j}, x_{j}\right)=v_{i}(t)+J_{i}+\sum_{j=1}^{n} w_{i j} \cdot e_{i j} \cdot x_{j}\left(t-l_{i j}\right)$

Here, $i, j=1,2, \ldots, n$, where $n$ corresponds to the total number of neurons. $x_{i}$ is the generated spike event (firing or no-firing) in a receiving neuron $i$, and $x_{j}\left(t-l_{i j}\right)$ is the state of a transmitting neuron $j$. The membrane potential is given by $f_{i}$, in which $J_{i}$ matches synaptic noise. The noise is independent for different neurons $i$. The nonlinear dynamics $v_{i}(t)$ of the neuronal membrane potential of neuron $i$ are defined by the Izhikevich model (Izhikevich 2003) with a simulation step $\mathrm{d} t=1 \mathrm{~ms}$. The neuronal input from the network, represented by the summation of postsynaptic pulses on the right-hand side of Eq. 1 is calculated with $e_{i j}=-1$ if $j$ belongs to the inhibitory neuronal pool and 1 otherwise; $l_{i j}$ is the latency between a spike on neuron $j$ and the postsynaptic potential on neuron $i$, and $w_{i j}$ denotes the corresponding synaptic weight. The percentage of excitatory neurons in the network was set to $R=80 \%$, as found in experimental studies, e.g., Toledo-Rodriguez et al. (2003). Values $l_{i j}$ were normally distributed between 1 and $D_{\max }$, the latter varying between 1 and $20 \mathrm{~ms}$ according to experimental findings in Muller et al. (1997). The number of connections per neuron followed a normal distribution between 0 and $K_{\text {max }}$, where $K_{\max }$ varied up to 1,000 in agreement with several experimental findings, e.g., Ichikawa et al. (1993). To mimic synaptic depression and facilitation affecting $w_{i j}$, we used a phenomenological short-term plasticity model (Markram et al. 1998). Together, all $w_{i, j}$ constituted a matrix $\mathrm{W}$ which consisted of values taken from a normal distribution between
0.01 and $9 \mathrm{mV}$ (Gibson and Connors 2003). Resuming, the just described adjustment of all basic parameters of the network model in experimental physiological ranges enables to directly relate experiment to simulation.

We studied bursting behavior in two types of network models. The first one had a normal distribution of all synaptic strengths; its average weight $\left(W_{\mathrm{e}}\right)$ was varied between 0.01 and $9 \mathrm{mV}$. In the second we chose a random subset of neurons in which the average synaptic weight to their targets ( $\left.W_{\text {int }}\right)$ was set higher than $W_{\mathrm{e}}$. Neurons from this subset are further referred to as intense neurons. All other parameters, as well as the network structure, remained unchanged.

One of the urgent issues and open questions in this field is the origin of the spontaneous activity. Possible sources are synaptic noise (Hubbard et al. 1967; Destexhe et al. 2004) or rhythmic pacemaker currents (Strata et al. 1997). The latter assumes the presence of intrinsically active cells (Latham et al. 2000). The former is based upon the probability of quantal release, randomness of diffusion, and chemical reaction, or on the unpredictability of responses from ion channels within the synaptic cleft (Mainen and Sejnowski 1995; Stevens and Zador 1998; Rodriguez-Molina et al. 2007). Vladimirski et al. (2008) showed that noise-driven, unstructured networks require large, nonphysiological values of synaptic parameters in order to produce robust network bursts, while structured networks can use lower values for the same purpose (Kitano and Fukai 2007). To trigger spontaneous activity we simulated both network types, i.e., noise- and pacemaker-driven networks. In the noise-driven networks each neuron received a Poissonian train of 1-ms pulses with amplitudes normally distributed between 0 and $6 \mathrm{mV}$ for excitatory and between 0 and $3 \mathrm{mV}$ for inhibitory neurons. The same method, validated by Destexhe et al. (2004), was used in Gritsun et al. (2010). The mean rate and variance were gradually increased from $F_{\mathrm{n}}=0$ to $F_{\mathrm{n}}=1 \mathrm{kHz}$, as suggested by Rodriguez-Molina et al. (2007). Similar approaches were also suggested in other studies, e.g., Nesse et al. (2008). In the pacemaker-driven simulations we kept all input parameters within the same physiological ranges as before, except that the noise was set at zero and pacemaker features were assigned to the intense neurons. We applied two methods to trigger intrinsic firing in the pacemaker neurons. First, we set a larger subthreshold current flux in the Izhikevich neuronal model as described in Gritsun et al. (2010). Without any external input those "pacemakers" could fire either periodic spikes or bursts of spikes with frequencies ranging up to $0.26 \mathrm{~Hz}$. In the second method, referred to as a "pseudo-pacemaker"-driven networks, instead of changing intrinsic neuronal features, we injected external pacemaker pulses into the subset of intense neurons. Intrinsic firing in the population of pacemakers was modulated by injecting 1-ms pulses. Injected pulses had the same amplitude per pacemaker (normally distributed between 0 and $6 \mathrm{mV}$ ), but an individual 
constant frequency $\left(F_{\mathrm{p}}\right)$. Pacemaker frequencies were chosen from a certain distribution such that evoked (intrinsic) firings resulted in biased ISI distributions in the whole pacemaker population. Individual $F_{\mathrm{p}}$ values were chosen from a generalized extreme value (GEV) distribution. Similar simulation results were obtained using other common distributions, such as Gamma or exponential distributions. The GEV distribution is the limit distribution of properly normalized maxima of a sequence of independent and identically distributed random variables (Coles 2001). The GEV probability density function is defined as follows:

$p_{\mathrm{GEV}}(x)=\frac{1}{\sigma} t(x)^{\xi+1} \mathrm{e}^{-t(x)}$,

where

$t(x)= \begin{cases}\mathrm{e}^{-(x-\mu) / \sigma} & \text { if } \quad \xi=0 \\ \left(1+\xi \frac{x-\mu}{\sigma}\right)^{-1 / \xi} & \text { if } \quad \xi \neq 0\end{cases}$

We tried a wide range of the shape $(\xi)$, scale $(\sigma)$, and location $(\mu)$ parameters to arrange the $F_{\mathrm{p}}$ distributions such that they had a frequency span from 0 up to $10 \mathrm{~Hz}$, and their shape varied from about exponential to almost normal. To obtain adequate pacemaker frequency distributions, particularly the location parameter $(\mu)$ was very important (i.e., to avoid near-zero frequencies which would reduce pacemakers to regular neurons). We also used the GEV distribution to characterize IBI distributions, as described later. Here we present the results of batch simulations in which the key parameters $\left(K_{\max }, W_{\mathrm{e}}, W_{\text {int }}, F_{\mathrm{n}}\right.$, and $F_{\mathrm{p}}$ set) were varied gradually one by one and the activity patterns were generated for $1 \mathrm{~h}$.

\subsection{Spiking activity: characterization and statistics}

The same characterization and statistical analysis were applied to both experimental and model data. For model analysis of IBIs we selected spike trains from 60 randomly chosen simulated neurons in the appropriate excitatory/inhibitory ratio (usually 48 excitatory plus 12 inhibitory). This allowed to compare with the experimental data, as the electrode arrays contain 60 electrodes.

Bursts were identified using a detection algorithm as described in Stegenga et al. (2008). In short, a network burst was detected when at least two spikes occurred within 10 -ms bins for each active electrode (with spike rate $>0.1 \mathrm{~Hz}$ ). Network bursts were characterized as follows: (1) Intraburst parameters were measured as described in a previous study (Gritsun et al. 2010); (2) IBIs were calculated as the temporal distances between peaks of neighboring bursts.

We recorded most of the cultures daily during several hours. IBI samples were taken from 1-h recordings. For histograms we used the bin size estimation by Scott (1979). This is given by $h=3.49 \cdot \mathrm{S} D \cdot N^{-1 / 3}$,

where $N$ is the number of IBIs in the sample, and SD is the IBI standard deviation.

We described IBIs using the median value as well as 16th and 84th percentiles, in order to show any bias in the distribution. The latter describe the $68 \%$ confidence interval, similar to \pm SD in normally distributed data. This representation does not always classify the data clearly and can be improved by adding the burst count distribution (BCD). To this end, we divided 1-h recordings into bins of $1 \mathrm{~min}$ and counted the number of bursts in each bin. BCDs were evaluated with mean and standard deviation, and we used burst counts mostly for the classification (grouping) of experimental samples. BCDs can show whether bursts occur regularly, randomly, or as burst clusters. Finally, under the assumption that IBIs are independent, identically distributed, and drawn from a particular distribution, we applied the method of maximum-likelihood estimation to identify the common distribution that fitted the grouped IBIs, with minimal error of the estimated parameters.

In addition, we characterized spike clustering regimes generated by the model using the Fano factor, as a dispersion index for spike counts. We divided 1-h recordings into 5 -ms bins and counted the number of spikes in each bin. The Fano factor for spike counts $\left(\mathrm{FF}_{\mathrm{s}}\right)($ Teich et al. 1984) is defined as the variance-to-mean ratio:

$\mathrm{FF}_{\mathrm{s}}=\sigma_{\mathrm{s}}^{2} / \mu_{\mathrm{s}}$.

Taking into account that typical burst widths range upwards from about $40 \mathrm{~ms}$, this time bin gives us $\geq 8$ data points. With $\mathrm{FF}_{\mathrm{s}}=1$, spikes follow a Poisson process and so $\mathrm{FF}_{\mathrm{S}}$ can be interpreted as the measure of spike asynchrony. Clusters of spikes, or in other words bursts, occur if $\mathrm{FF}_{\mathrm{S}}>1$. Furthermore, the firing behavior of models can be characterized by the sensitivity of $\mathrm{FF}_{\mathrm{s}}$ to one of the gradually changing parameters, in particular the noise frequency $F_{\mathrm{n}}$. In this article we present three operational regimes of noisedriven network models, depending on $\mathrm{FF}_{\mathrm{S}}$ and its sensitivity to noise frequency changes. If $\mathrm{FF}_{\mathrm{S}}=1$ and insensitive to changes of $F_{\mathrm{n}}$ we will speak of asynchronous spiking. If $\mathrm{FF}_{\mathrm{s}}>1$ and if $\mathrm{FF}_{\mathrm{s}}$ is highly sensitive to $F_{\mathrm{n}}$ changes, we will classify the operational regime as weakly synchronized, or as completely synchronized if $\mathrm{FF}_{\mathrm{s}}$ is insensitive to $F_{\mathrm{n}}$ changes.

\section{Results}

\subsection{Statistics of experimental data}

The experimental data were collected from 13 cultures, recorded between 2 and 11 weeks in vitro with a total of 

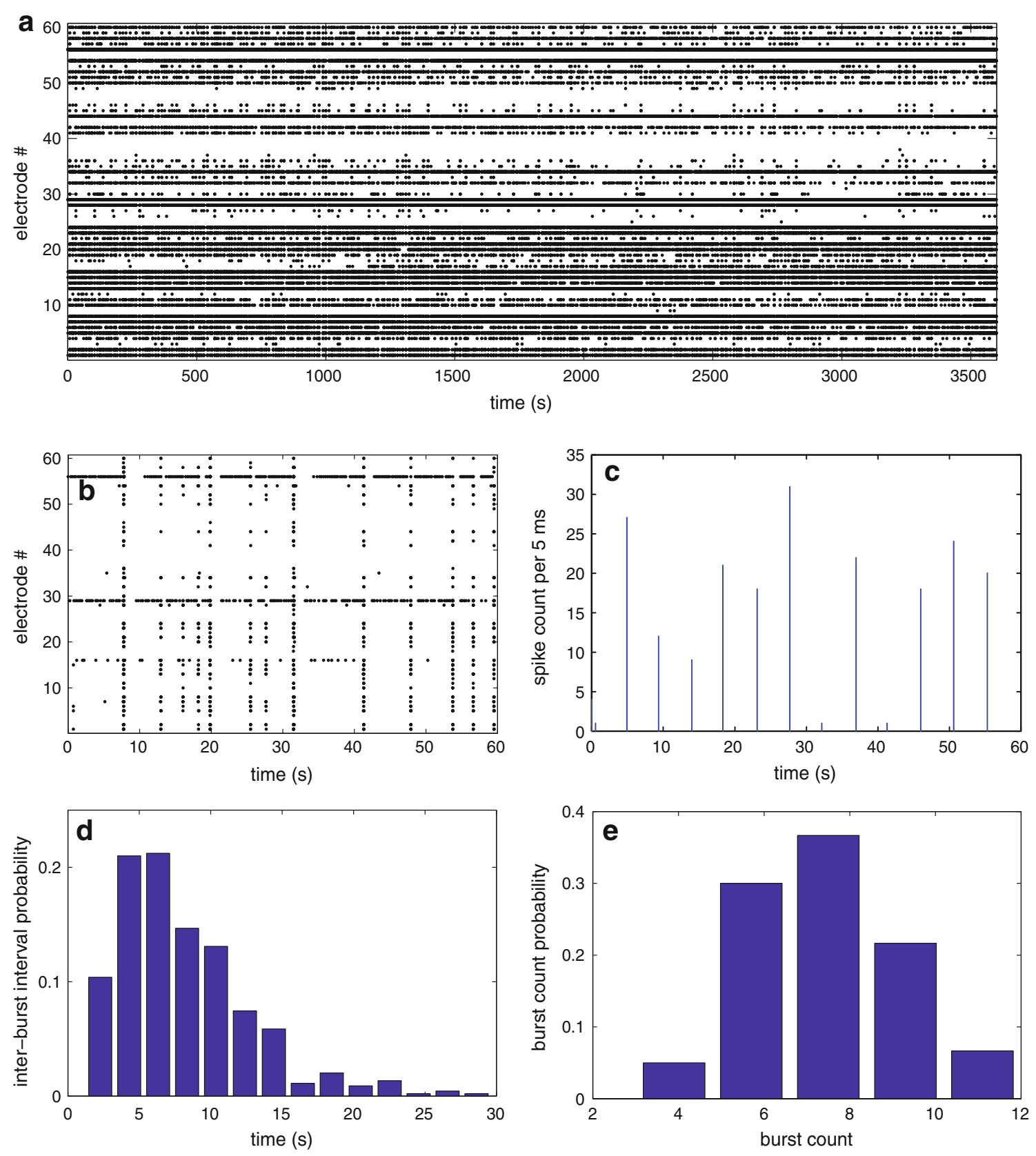

Fig. 2 Example of experimental data sample acquired from culture \#5, 22 DIV. a Spike raster of a complete $1 \mathrm{~h}$ sample. Each dot represents a detected action potential at the indicated electrode. b Zoomed spike raster of the first minute of a. $\mathbf{c}$ To calculate spike counts, the data from b are binned into 5-ms intervals and summed. If the joint spike count of any two consecutive bins exceeded two times the number of active

electrodes, these bins were marked as a burst (see Sect. 2.3). Then, we calculated the length of all intervals between the detected bursts (IBIs). d shows the IBI distribution. Finally, we counted the number of bursts in 1-min intervals. e shows the BCD. The histograms in $\mathbf{d}$ and $\mathbf{e}$ were calculated from the data in a

206 recorded hours (samples) containing 61,343 bursts. All recorded samples had $\mathrm{FF}_{s} \mathrm{~s}$ ranging from 5 to 70, indicating weakly or completely synchronized regimes. To begin, we illustrate the characterization using a typical example of bursting activity acquired from one of the cultures. Bursting behavior is basically represented as spike clusters throughout all recorded neurons (electrodes). Figure 2a, b shows spike raster examples of $1 \mathrm{~h}$ and $60 \mathrm{~s}$, respectively, acquired from culture \#5 at 22 days in vitro (DIV). One can notice synchronized firing recorded from most of the electrodes in Fig. 2b, which is also indicated by the spike counts shown in Fig. 2c. Bursts seemed to occur in small 
clusters, which gave higher occurrence of short IBIs than long ones. This in turn led to skewed IBI distributions (Fig. 2d). The relative burst frequency for this particular example ranged from 3 to 12 bursts per minute, as shown in Fig. 2e.

We analyzed IBIs in recordings that lasted from 1 to $27 \mathrm{~h}$ in cultures of different age. In longer recordings $(>3 \mathrm{~h})$ of individual cultures this analysis showed stable median and 16th and 84th percentiles values within at least $3 \mathrm{~h}$, and in some cases this stability was observed during the whole recorded period.

Next, we pooled all samples and analyzed their overall distribution to show the data range over all recordings.

The histogram in Fig. 3a corresponds to the relative frequency distribution of the number of IBIs over all recorded samples. This IBI histogram shows a highly skewed distribution with a median of $4 \mathrm{~s}$ and a long tail, scattered up to several minutes. Using Eq. 2 we obtained the 16th and 84th percentiles, resulting in a $68 \%$ confidence interval between 1.5 and $17.5 \mathrm{~s}$. As the IBI distribution turned out to be a smooth histogram, it follows that the burst rate had a smooth distribution ranging from $1 \mathrm{~Hz}$ down to near-zero values.

Figure $3 \mathrm{~b}$ shows the relative distribution of bursts counts, for all recorded samples. The interval from the global peak at $1 \mathrm{burst} / \mathrm{min}$ to the leftmost local minimum around 8 bursts/ min reflects long IBIs (the long tail in IBI histogram in Fig. 3a). This interval is followed by a BCD showing two local maxima around 13 and 28 bursts/min, which reveal two frequency ranges of burst clusters: one around $0.22 \mathrm{~Hz}$ and the second around $0.47 \mathrm{~Hz}$.

Median and percentile ranges of the pooled data showed much lower values than in individual samples, which typically had a median spanning from 1.7 to about $35 \mathrm{~s}$ and a $68 \%$ confidence interval between 1 and $85 \mathrm{~s}$. Such large span variability hampers critical model validation. Therefore, we divided all samples into several groups. A few samples $(<5 \%)$ with quite large data spans (thus having scarce data points) were not used further. This grouping helped to obtain an ample amount of data for more precise estimation of probability density functions.

The following method was used for grouping. The mean of the burst counts per minute in 1-h samples was calculated (Fig. 4a). We divided the samples according to their burst count mean values as shown in Fig. 4a, where each color corresponds to a different group. Figure $4 \mathrm{~b}$, c shows IBI (top) and BCD (bottom) histograms, respectively, for each group. Table 1 summarizes the grouped experimental data: median and \pm percentiles for IBI distributions and mean $( \pm \mathrm{SD})$ for BCDs. To find a common distribution fitting all four groups with minimum error, we tried several distributions, including Gamma, Gaussian, GEV, exponential, lognormal (scale-free), etc. The GEV distribution gave the best fit with the smallest error of the estimated parameters for all groups (Table 1). IBIs acquired from subsequent samples (recorded hours) of an individual culture always ended up in the same group.
Fig. 3 Overview of the interburst parameters over all recordings. a IBI distribution. Bin size was calculated using Eq. 3. b BCD. We counted the number of bursts in each 60 -s interval and plotted the distribution of burst counts
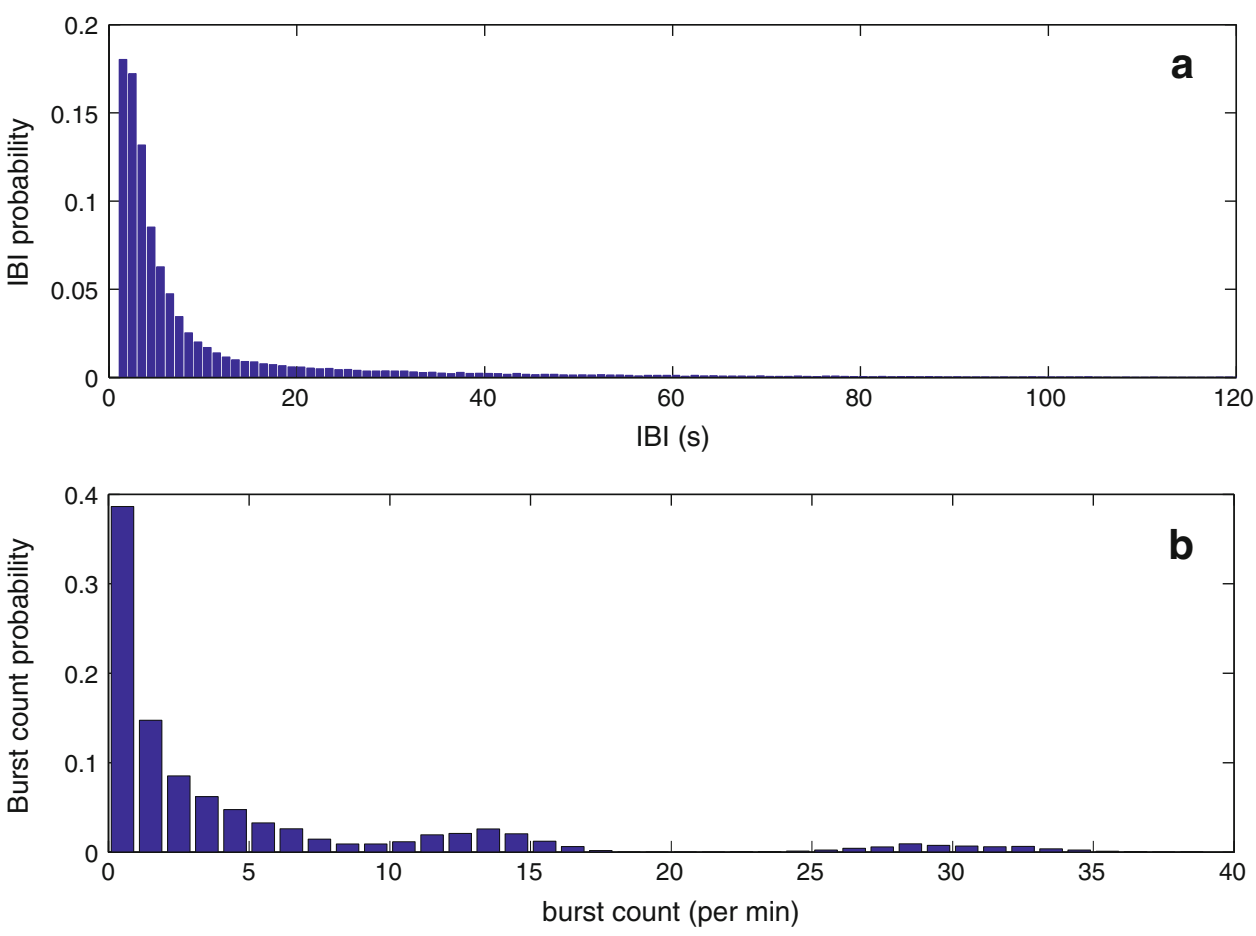
Fig. 4 Sample grouping of experimental data according to burst count means. a In each 1-h recording sample we counted the number of bursts per 1-min interval and calculated the mean. The histogram shows the number of samples with the specified mean burst count. Then we divided the data into four groups; each group is depicted with a different color. b, c IBI distributions (top) and BCD histograms (bottom) for the four groups

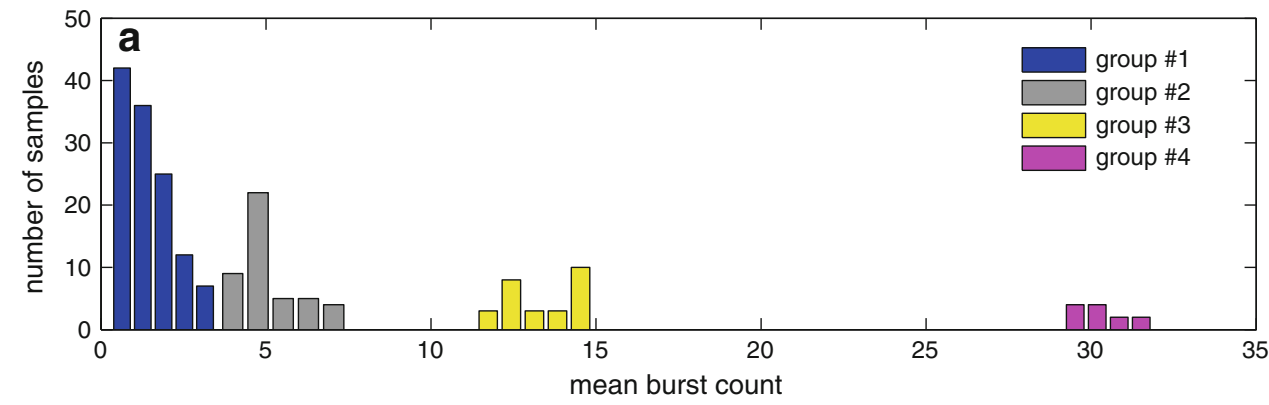

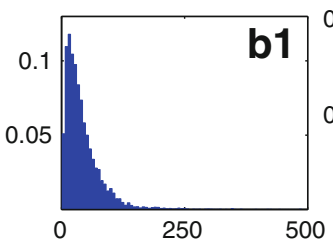

IBI (s)

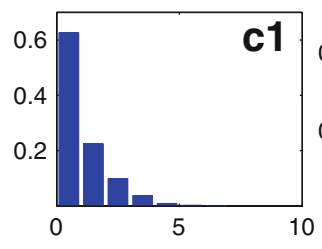

burst count

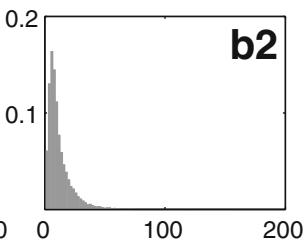

IBI (s)

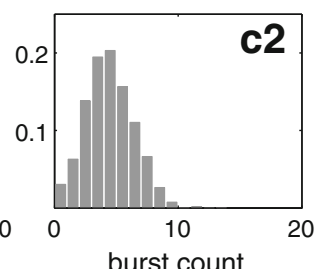

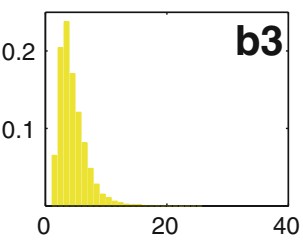

IBI (s)

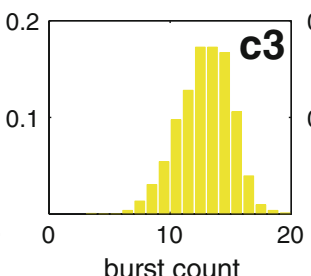

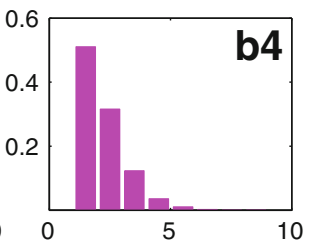

IBI (s)

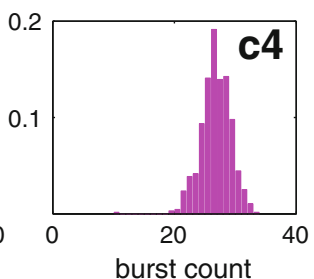

Table 1 Statistics summary for the grouped experimental IBIS and BCDs

\begin{tabular}{|c|c|c|c|c|c|}
\hline \multicolumn{6}{|l|}{ General } \\
\hline Group number & 1 & 2 & 3 & 4 & Total \\
\hline \# Samples & 122 & 45 & 27 & 12 & 206 \\
\hline \# Network bursts & 9617 & 12359 & 20969 & 18395 & 61340 \\
\hline \multicolumn{6}{|l|}{ Interburst interval statistics } \\
\hline Median & 32.3 & 9 & 4 & 2 & 4.15 \\
\hline Upper/lower percentiles & $71.65 / 13.0$ & $19.6 / 4.57$ & $6.44 / 2.53$ & $3.08 / 1.32$ & $17.41 / 1.89$ \\
\hline$\mu(95 \% \text { confidence interval })^{\mathrm{a}}$ & $24.2(0.22)$ & $7.23(0.05)$ & $3.4(0.012)$ & $1.72(0.005)$ & \\
\hline 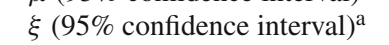 & $0.35(0.01)$ & $0.33(0.008)$ & $0.14(0.005)$ & $0.2(0.008)$ & \\
\hline$\sigma(95 \% \text { confidence interval })^{\mathrm{a}}$ & $18.43(0.19)$ & $0.33(0.008)$ & $1.5(0.009)$ & $0.6(0.004)$ & \\
\hline \multicolumn{6}{|l|}{ Burst count distribution } \\
\hline Mean & 1.4 & 4.95 & 13.36 & 27.3 & 5.28 \\
\hline Standard deviation & 1.1 & 1.95 & 2.18 & 2.48 & 6.96 \\
\hline
\end{tabular}

${ }^{\text {a }}$ Parameters estimated for a data fit to the GEV distribution, using the method of maximum-likelihood estimates (and 95\% confidence intervals for the parameter estimates)

3.2 Noise-driven simulations; model response to increasing synaptic noise

\subsubsection{Network simulation with homogeneous synaptic strength distribution}

As a basic model check, we examined how the neuronal population responded to the injected noise only, i.e., without any synaptic connections (interactions). We found that the network-wide ISI decreased exponentially with the average noise frequency, both in the excitatory and in the inhibitory population (data not shown). After this basic check, simulations were done where network parameters were set as described in "Methods." Figure 5 shows a typical example of a simulation with physiologically plausible network parameter values: $K_{\max }=500, D_{\max }=10 \mathrm{~ms}$, $R=80 \%, W=1.5 \mathrm{mV}$. We kept all model parameters, except the network size, in a constant range in order to have a common point of reference. For small networks created with this setup, parameter ranges were similar to those reported in literature, e.g., Wiedemann and Luthi (2003), Giugliano et al. (2004), and Nesse et al. (2008).

By changing the network size from $n=500$ to 5,000 and keeping $K_{\max }$ constant, the average number of connections 
Fig. 5 Four sensitivity plots of the Fano factor of spikes $\left(\mathrm{FF}_{\mathrm{s}}\right)$ in response to gradually increasing noise frequency $\left(F_{\mathrm{n}}\right)$ for networks of different size $n$, in three synchronization regimes. Dashed curves correspond to weakly synchronized case $(n=500$, 2,500 , and 4,000 ). Note the sudden change from asynchronous to completely synchronized for $n=5,000$

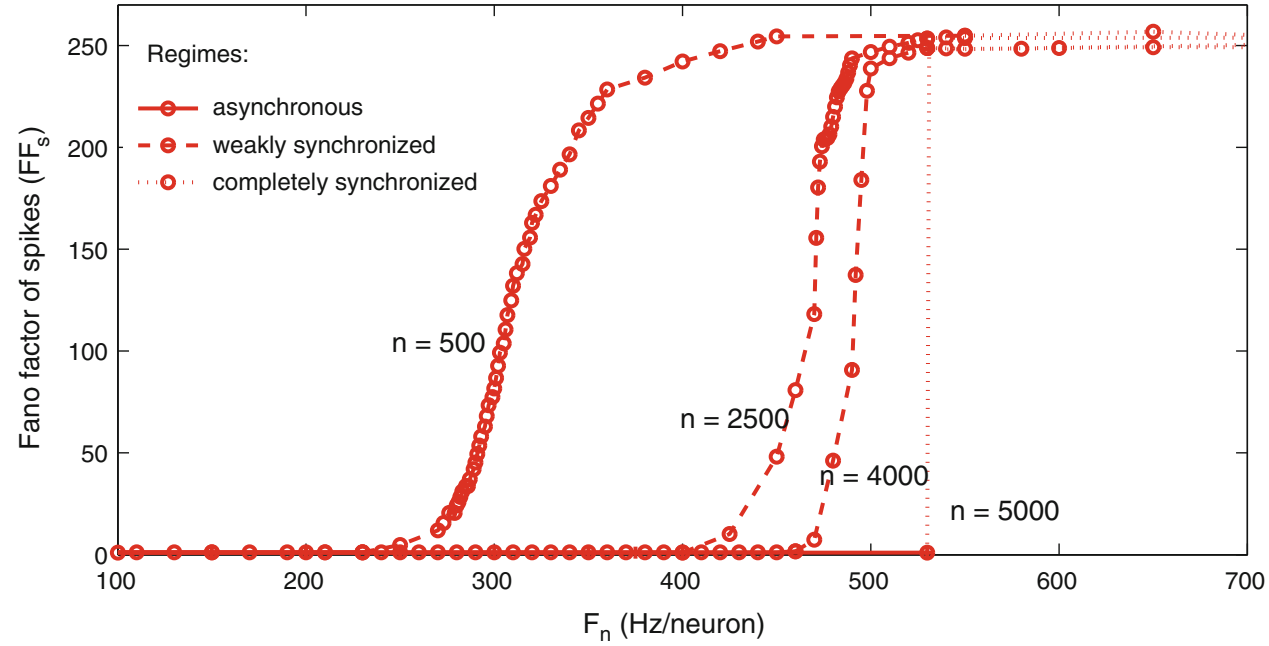

(or "average connection probability") between two randomly chosen neurons was reduced from 1 to 0.1 . This can be understood as follows. The number of unique postsynaptic neurons to which a presynaptic neuron is connected increases with the network size, as every presynaptic neuron has more possible postsynaptic neurons to be connected to. Conversely, this number of unique neurons is reduced in smaller networks, because every presynaptic neuron is more likely to have multiple connections to the same postsynaptic neuron.

We analyzed network behavior in response to gradually increasing synaptic noise in batch simulations with a simulated duration of $1 \mathrm{~h}$. We observed three major operational regimes in these networks.

At low noise frequencies $\left(F_{\mathrm{n}}\right)$, networks responded with asynchronous spikes and their population firing rate increased proportionally to $F_{\mathrm{n}}$. This regime is further referred to as asynchronous spiking and was characterized by $\mathrm{FF}_{\mathrm{s}}=1$ and the absence of network bursts. Thus, spiking behavior of networks with low noise frequencies could be characterized as a Poissonian process. With increasing noise frequency all networks eventually produced activity with ISIs that were short enough to produce synchronized behavior, which would then propagate throughout the whole neuronal population. Relatively small networks with a small number of unique postsynaptic neurons usually elicited irregular bursts. Burst frequency increased with higher $F_{\mathrm{n}}$. A linear increase of $F_{\mathrm{n}}$ resulted in a sigmoid increase of $\mathrm{FF}_{S}$ while the median IBI reduced exponentially. At relatively high noise frequencies ( $F_{\mathrm{n}} \geq 530 \mathrm{~Hz} /$ neuron) this bursting regime reached the saturation point where networks produced high-frequency bursts in a more regular manner, as expressed by a stable (saturated) $\mathrm{FF}_{\mathrm{s}}$. At such high $F_{\mathrm{n}}$, IBIs reached a stable value of less than $1 \mathrm{~s}$, while $\mathrm{FF}_{\mathrm{s}}$ reached a plateau (around 250 for this specific setup). We differentiated between two regimes of bursting behavior: weakly synchronized and completely synchronized regimes, yielding irregular and regular bursts, respectively.

Small networks in the weakly synchronized regime produced irregular bursts with IBI distributions similar to the experimental ones. The range of $F_{\mathrm{n}}$ that yielded weakly synchronized activity with IBIs as in the experimental range shortened with increasing network size. We found that large networks $(n=5,000)$ switched instantly from asynchronous spiking to a completely synchronized regime, without an intermediate regime of weakly synchronized activity, as shown in Fig. 5. At $F_{\mathrm{n}} \approx 530 \mathrm{~Hz} /$ neuron networks abruptly switched from random spiking to highly ("super") regular bursts (by increasing $F_{\mathrm{n}}$ only by $1 \mathrm{~Hz}$ ). Furthermore, analysis of the intraburst parameters showed that these simulations produced unnaturally long and robust bursts with higher maximum firing rate than experimentally observed.

\subsubsection{Network simulation with bimodal distribution of synaptic strengths}

The number of neurons in cultures is much larger than $n=5,000$, but cultures do not exhibit the just observed "super" regular bursting as the simulations in the previous section. To find a more realistic bursting activity in large networks, we analyzed the behavior of models with $n=5,000$ and the same parameters as in the previous section, but now with a subpopulation of intense neurons $\left(n_{\text {int }}=250\right)$. Intensity variation means that we gradually increased the synaptic weights of connections that project from the intense neurons. Stronger synapses directly increase network feedback, so these neurons should now need less injected noise to produce bursting. We set the noise frequency relatively low (to $F_{\mathrm{n}}=220 \mathrm{~Hz} /$ neuron). Then, we 
Fig. 6 Sensitivity curve of the Fano factor of spikes $\left(\mathrm{FF}_{\mathrm{s}}\right)$ to a changing synaptic strength ratio of intense and regular neurons $\left(W_{\text {int }} / W_{\mathrm{e}}\right)$. Networks change their behavior from to bursting $\left(\mathrm{FF}_{\mathrm{S}}>1\right)$ around $W_{\text {int }} / W_{\mathrm{e}}=65$ asynchronous spiking $\left(\mathrm{FF}_{\mathrm{S}}=1\right)$

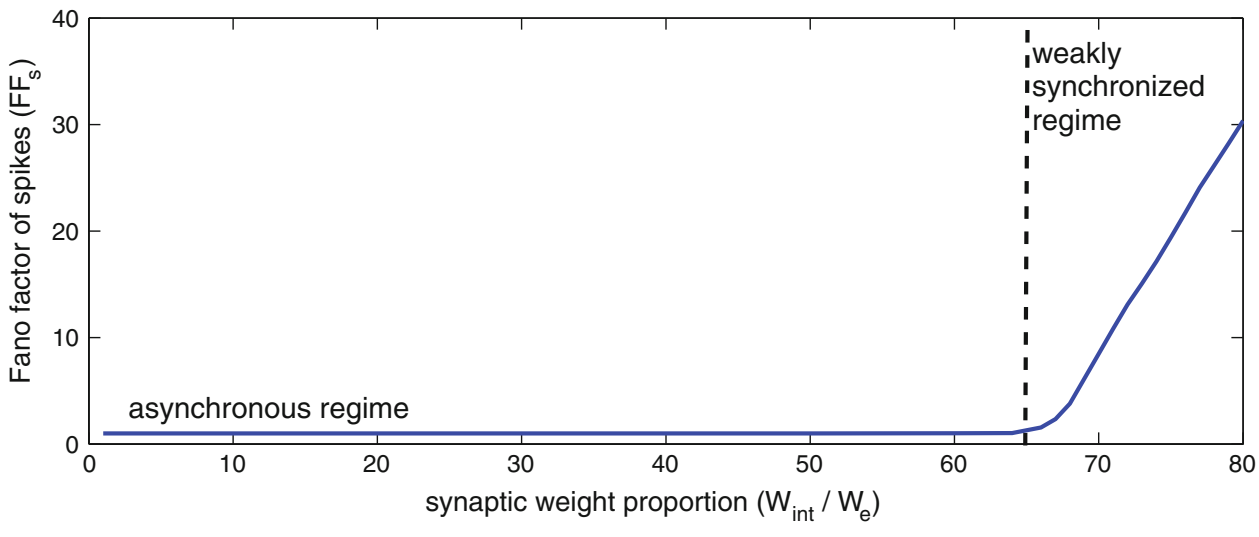

gradually increased the average synaptic strength of intense neurons $\left(W_{\text {int }}\right)$ and proportionally reduced the amplitude of synaptic strength in all other neurons. We extended the range of $W_{\text {int }}$ to $9 \mathrm{mV}$, whereas the synaptic strength of regular neurons $\left(W_{\mathrm{e}}\right)$ ranged from 0.01 to $0.1 \mathrm{mV}$. Figure 6 shows how the $\mathrm{FF}_{\mathrm{s}}$ depended on the synaptic strength ratio $\left(W_{\text {int }} / W_{\mathrm{e}}\right)$.

Network behavior now changed from asynchronous spiking to a weakly synchronized regime around $W_{\text {int }} / W_{\mathrm{e}}=65$. In this weakly synchronized regime, networks produced stable random bursting throughout the entire simulation time. The resulting $\mathrm{FF}_{\mathrm{s}}$ resembled the experimental range. Moreover, network models in this operational regime showed lower sensitivity to changing network parameters than networks with homogeneous synaptic strengths. In the following example we set the $\left(W_{\text {int }} / W_{\mathrm{e}}\right)$ ratio to 73 , while the network was exposed to synaptic noise with increasing frequency. Figure 7 shows a typical example of network activity at a certain noise frequency, as well as the development of $\mathrm{FF}_{\mathrm{S}}$ in response to gradually increasing noise frequency. With a bimodal distribution of synaptic strengths, bursting appeared irregularly and $\mathrm{FF}_{\mathrm{s}}$ increased smoothly. Similar results were obtained with other combinations of network parameters. Thus, these networks generated a wider range of synchronization, leading to more realistic patterns of spiking activity.

Figure 8 shows how the range of IBIs changed during noise frequency sweeps. IBI now smoothly decreases with increasing $F_{\mathrm{n}}$, and it is easy to reproduce all four experimentally found ranges of IBIs. More particularly, comparison of these values in Fig. 8a with those in Table 1 shows that simulations with $F_{\mathrm{n}} \approx[225,255,295$, and 330$] \mathrm{Hz}$ resemble the experimental groups \#1 to \#4, respectively.

To clarify the origin of bursts in a weakly synchronized regime we investigated the spikes that preceded a burst. In most cases the intense neurons fired first, thereby triggering an avalanche effect of network activity. As one would expect, networks responded to firing of the intense neurons because they were able to produce strong excitatory input

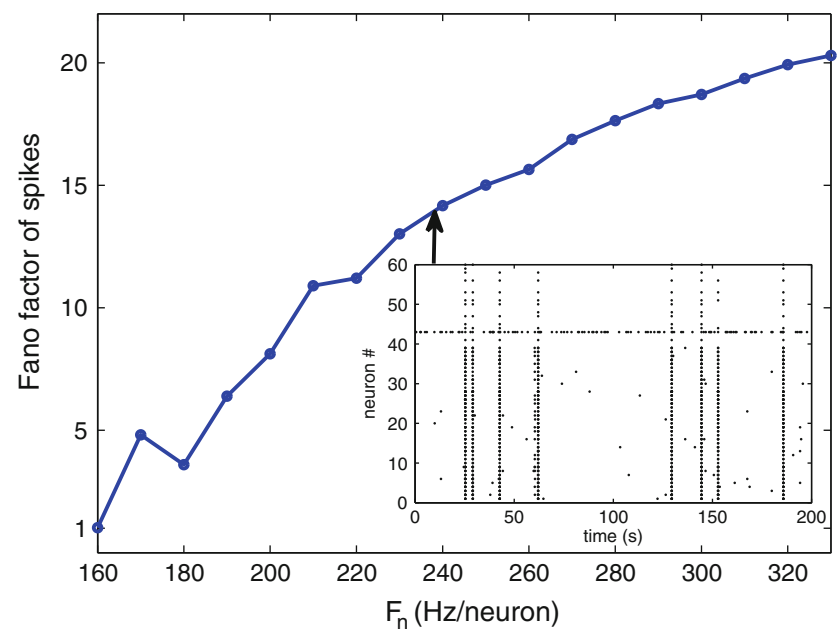

Fig. 7 Example of the Fano factor of spikes $\left(\mathrm{FF}_{\mathrm{s}}\right)$ as a function of the synaptic noise frequency $\left(F_{\mathrm{n}}\right)$ in a network with a bimodal distribution of synaptic strengths. In this example we set $W_{\text {int }} / W_{\mathrm{e}}=73$. Inset example of a spike raster simulated at a noise frequency $F_{\mathrm{n}}=240 \mathrm{~Hz} /$ neuron as indicated by the arrow

to the network through their stronger synapses. We recall that, with our set of neuronal parameters, noise-evoked spiking activity typically had an exponential ISI distribution. We note that IBI distributions were proportional to the ISI distributions of noise-evoked action potentials in the subpopulation of intense neurons. Furthermore, the sensitivity curve in Fig. 8a suggests that the IBI range is inversely proportional to the injected noise frequency. However, this relationship is nonlinear: with increasing $F_{\mathrm{n}}$ the average bursting frequency increases progressively (Fig. 8b). This is due to a higher overall neuronal excitability because higher $F_{\mathrm{n}}$ induces higher average neuronal membrane potential in the whole network (data not shown).

This network model describes bursting behavior quite well. Still, several studies suggest the presence of endogenously active cells (Sect. 2). Therefore, we also investigated pacemaker-driven networks. 

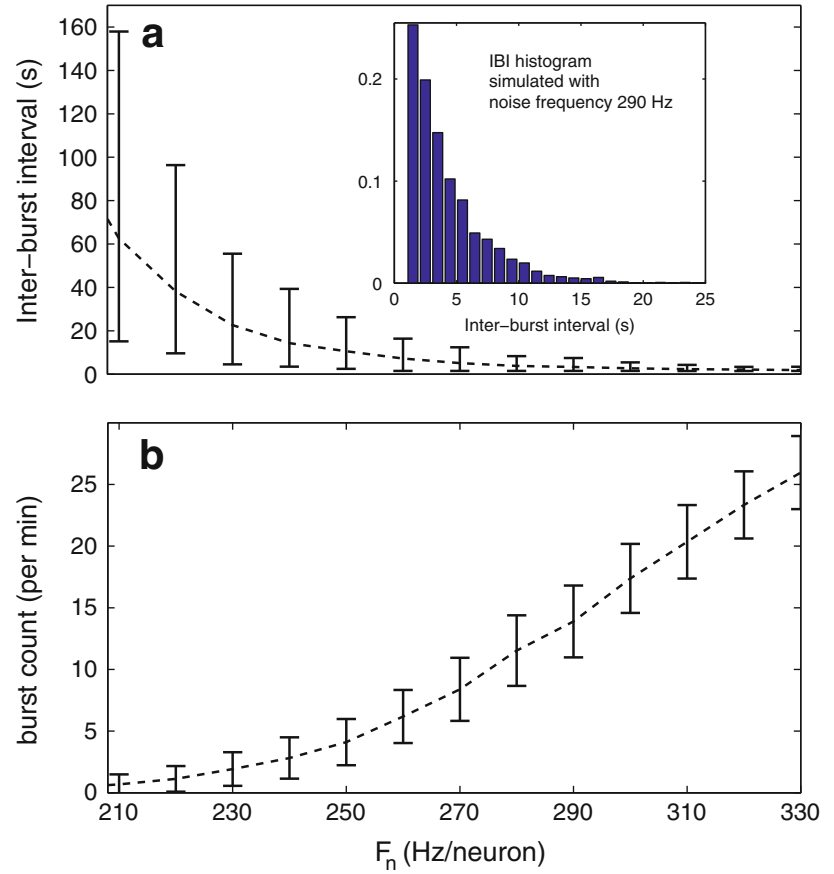

Fig. 8 Sensitivity plots for interburst parameters in response to increasing noise frequency $\left(F_{\mathrm{n}}\right)$ in network models with bimodally distributed synaptic strengths. a IBI median (dashed line) and range (16th-84th percentiles, bars). Inset example of an IBI histogram at $F_{\mathrm{n}}=290 \mathrm{~Hz}$. b Burst count mean (dashed line) and standard deviation (bars)

\subsection{Pacemaker-driven networks}

In this part we briefly describe the activity of two network models with a small population of intense neurons having pacemaker features, as described in Sect. 2. This setup was based on the assumption that intrinsically active neurons may develop strong synapses through long-term potentiation processes while initiating activity in neighboring neurons at early developmental stages of a neuronal culture. While this hypothesis is yet to be proven experimentally, we achieved successful simulation results.

In the models where intrinsic (pacemaker) firing was triggered by large current influx, bursts were initiated by the pacemakers with the fastest oscillations [while discharging all other (slower) pacemakers during the generated network burst]. Therefore, these simulations only produced periodic bursts with constant IBIs, which were fully determined by the frequency of the fastest pacemaker (data not shown).

In the pseudo-pacemaker-driven model, instead of changing neuronal features, we injected external pacemaker pulses into the subset of intense neurons. Thus, even if a pacemaker was discharged by the previous burst, it could fire again in response to these external pulses and therefore trigger its own bursts series. These simulations generated irregular IBIs, as shown in Fig. 9 for a few typical examples of IBI distributions

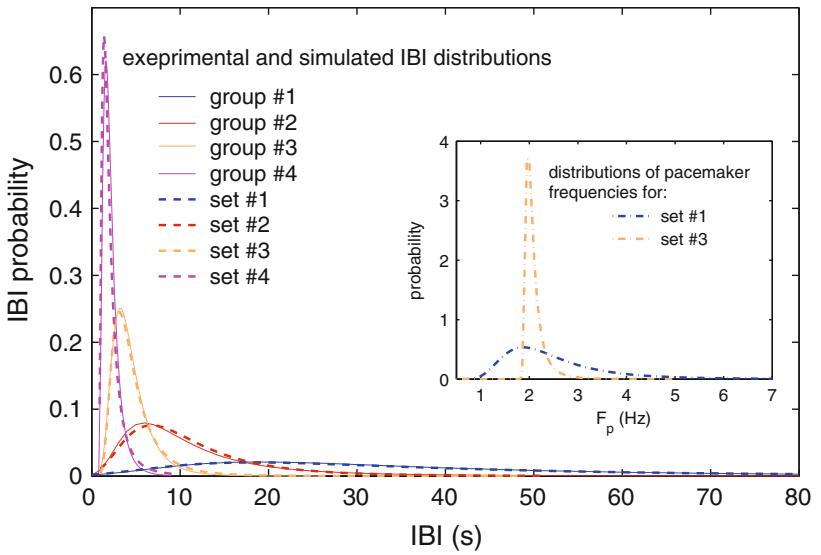

Fig. 9 IBI distributions of experimental data (groups) and simulated with pseudo-pacemaker-driven networks (sets). The inset shows the distributions of $F_{\mathrm{p}}$ to obtain the IBI distributions of sets \#1 and \#3. Simulation set numbers correspond to sets presented in Table 2

Table 2 Input parameter settings for simulations presented in Fig. 8

\begin{tabular}{lllll}
\hline Set \# & 1 & 2 & 3 & 4 \\
\hline$K_{\max }$ & 650 & 700 & 650 & 550 \\
$W_{\text {pr }} / W_{\mathrm{e}}$ & 11 & 12 & 10.7 & 13.5 \\
$N_{\text {intense }}$ & 250 & 100 & 250 & 150 \\
$\xi$ & 0.2 & 0.2 & 0.35 & 0.35 \\
$\sigma$ & 0.7 & 0.1 & 0.1 & 0.1 \\
$\mu$ & 2 & 2.4 & 2 & 2.7 \\
\hline
\end{tabular}

The $F_{\mathrm{p}}$ were chosen from the GEV distribution with shape $(\xi)$, scale $(\sigma)$, and location $(\mu)$ parameters as specified in the table. For all sets $n=5,000, D_{\max }=10 \mathrm{~ms}, R=80 \%$

that resembled the four groups of the experimental IBIs. In these simulations, the $F_{\mathrm{p}}$ were chosen from the GEV distributions as shown in Fig. 9 (inset) for sets \#1 and 3. Essential model parameter sets to generate these IBIs are presented in Table 2.

For all four network model sets, the GEV distribution gave the best fit to the simulated IBIs. Intraburst parameters were also verified to be in the experimental range (data not shown). The basic idea behind simulation of this realistic bursting behavior was that generated ISIs in a pacemaker population (set by adjusted intrinsic frequencies) triggered IBIs with distributions in the physiological range.

\section{Discussion}

\subsection{Experimental data}

Spontaneous activity in cultured cortical networks on multielectrode arrays has been studied by many experimenters 
(Van Pelt et al. 2004; Eytan and Marom 2006; Wagenaar et al. 2006; Stegenga et al. 2008). Networks typically show episodes of highly synchronized spiking, commonly referred to as network bursting. $\mathrm{FF}_{\mathrm{S}}$ values in all recorded samples were above 1 and well below the values generated by simulations in completely synchronized regimes $\left(\mathrm{FF}_{\mathrm{s}} \approx 250\right)$, thus suggesting that cultured cortical networks operate in a weakly synchronized regime. Bursts are usually characterized by intra- and interburst parameters. In this study we focused on interburst parameters. All experimental recordings showed network bursts with a wide IBI variability, in agreement with earlier results found by Wagenaar et al. (2006), also suggesting a weakly synchronized regime. Segev et al. (2002) showed that experimental IBIs usually follow a scale-free (Levy) distribution. This finding was confirmed by the pooled data analysis in this study. However, individual experimental samples often showed multimodal distributions of IBIs. Multimodality in acquired data distributions might reflect a typical characteristic in the recorded firing patterns (e.g., in "superbursts"). However, experimental data containing relatively long IBIs ( $>30 \mathrm{~s}$ ) always appeared with multimodal distributions, whereas recordings with short IBIs usually yielded unimodal histograms. Obviously, (1-h) recordings with long IBIs yielded distributions based on fewer data points, suggesting that multimodal IBI distributions may mainly result from a relatively small amount of data. Most of the individual samples ( $>95 \%$ ) showed regular IBIs, whereas the pooled data were irregular and could not serve to validate our models. Based on the average burst frequency, all available recordings were divided into four representative groups, each showing regular IBIs (similar to the individual recordings). As a result, even the groups with ample data points showed smooth histograms. Thus, the grouped data characterized four ranges of interburst probabilities and were used to validate our models. It should be emphasized that this grouping was done to enable more critical model validation, rather than to describe distinct patterns of network activity which might be related to different physiological conditions. Additional data might have yielded a different set of groups, but with similar parameter ranges.

In summary, on the basis of $\mathrm{FF}_{\mathrm{S}}$ range and IBIs we concluded that cultured cortical networks operate in a weakly synchronized regime. Experimental IBIs, grouped according to their mean burst counts yielded smooth histograms, which we described by fitted GEV distributions.

\subsection{Simulated data}

For this study we simulated spiking activity in networks with 500-5,000 neurons. We evaluated the level of asynchrony (as defined by the stability of $\mathrm{FF}_{\mathrm{s}}$ ) for various combinations of network size and noise frequency. Only for networks operating in the weakly synchronized regime did small noise frequency changes significantly affect $\mathrm{FF}_{\mathrm{S}}$, whereas asynchronous and completely synchronized spiking regimes were insensitive to such changes. We found that only networks in the weakly synchronized regime could produce IBIs in realistic ranges.

Other studies showed that simulated activity in small networks resembled experimental data (Segev et al. 2001; Wiedemann and Luthi 2003; Giugliano et al. 2004). Giugliano et al. (2004) used relatively small networks $(n=100-1,000)$ with connection probability of $0.3-0.4$, and described three operational regimes, similar to ours. In their study, all three regimes occurred, and the effective regime was determined mainly by the set of synaptic weights. However, our study shows that, in large networks, with lower connection probability (but realistic in terms of average number of connections per neuron), the transition from a weakly synchronized regime to a completely synchronized one occurs upon a very small increase of the noise frequency. The main difference between small and large networks is their difference in connection probability. With similar $K_{\max }$, the probability that two randomly chosen neurons are connected to each other decreases in larger networks. On the one hand, Nesse et al. (2008) showed that, in fully connected networks of 500 neurons, increased noise could trigger an abrupt change in network activity from asynchronous to synchronized. On the other hand, Giugliano et al. (2004) showed that sparse networks can operate in a more realistic synchronized regime when the strength of synaptic interactions is adjusted. In fact, in developing cultured networks, the average number of synapses as well as the average synaptic strength per neuron increases in the first few weeks, (Ichikawa et al. 1993; Maeda et al. 1995). A higher connection probability results in synchronized network activity at lower noise frequencies. This widens the range of noise frequencies that yield synchronized activity. With lower connection probability, synchronized activity first occurs at much higher noise frequencies, and then tends to develop into complete synchronization immediately. In relatively large networks of 5,000 neurons, the weakly synchronized regime completely vanished. In such models we found either asynchronous spiking or completely synchronized activity, contradictory to the experimental data. Moreover, these noise-driven networks with homogeneous synaptic strengths could produce only regular bursts with IBIs shorter than $1 \mathrm{~s}$ (superbursts, Wagenaar et al. 2006). In a previous study, we found that such networks also showed unrealistic intraburst characteristics (Gritsun et al. 2010). Thus, simulated activity of noise-driven larger networks may not resemble experimental data at all, considering the fact that most data were recorded from much larger cultures containing from several thousand to around half a million neurons, e.g., Wagenaar et al. (2006), Van Pelt et al. (2004), and Stegenga et al. (2008). 
Experimental studies on cortical networks provide ample evidence for the existence of a special set of self-firing neurons that precede, or even ignite, network bursts as described in the "Introduction," e.g., privileged neurons (Eytan and Marom 2006), endogenously active neurons (Latham et al. 2000), and leader neurons (Eckmann et al. 2008). Eckmann et al. suggested three possible scenarios for how the leader neurons could be excited (Eckmann et al. 2008). In the first two scenarios, one or several leader neurons are self-excited or stimulated by low-level noise (in parallel) while exciting a region around them and randomly igniting network bursts. In their most likely scenario the subnetwork of the (noise-activated) leader neurons communicate among themselves while initiating network bursts. For simulations presented in this article we adopted two possible scenarios without disproving either of them (i.e., noise- and pacemaker-driven activity) as described in "Methods." However, the distinctive feature of our model is the bimodal distribution of synaptic strengths which implies a stronger subnetwork or circuit of intense neurons with relatively large synaptic outputs to the rest of the neurons. We showed that a subset of intense neurons in the network model largely increased the noise frequency range that yielded a weakly synchronized regime. Moreover, in recordings we often observed neurons with more or less continuously ongoing activity (Fig. 2a, b). These neurons preceded the network bursts always by less than $30 \mathrm{~ms}$ (Gritsun et al. 2010). Intracellular experiments showed that presynaptic spikes that precede postsynaptic firing by up to $\sim 30 \mathrm{~ms}$ lead to long-term potentiation of the connecting synapse (Bi and Poo 1998; Zhang et al. 1998; Song et al. 2000). Functional connections between neurons that have a latency in this range also tend to become strengthened in spontaneously active cultured cortical networks (le Feber et al. 2007, 2009). This adds justification to our assumption that neurons with ongoing activity have relatively strong synapses (intense neurons). Intense neurons triggered other neurons in a one-tomany fashion, whereas many regular neurons had to integrate their output to trigger another neuron. Thus, bursts were far more likely to be initiated by intense neurons than by regular ones. This activity triggering mechanism is somewhat similar to a two-layer synfire chain reaction where synchronous firing of intense neurons elicits synchronous volleys of spikes in regular neurons (but the synfire model lacks a realistic latency distribution among the neurons). Conversely, in networks with homogeneous synaptic strengths, any excitatory neuron could initiate a burst (depending on the average synaptic strength and/or noise frequency), resulting in a high burst probability. Therefore, by choosing a relatively small population of intense neurons, the bimodal network could generate bursts with a much lower IBI probability than the network with homogeneous synaptic strengths. Amit and Brunel (1997) showed that neurons with normally distributed input noise generated exponentially distributed
ISIs, which was confirmed in our simulations. Exponentially distributed ISIs in the set of intense neurons yielded exponentially distributed IBIs in turn, corresponding to weakly synchronized activity. It should be noted that most simulations with bimodal weights produced bursts with peak firing rates far below the maximum possible value, as seen in other experimental studies (Van Pelt et al. 2004; Eytan and Marom 2006; Wagenaar et al. 2006; Gritsun et al. 2010). This means that networks did not completely synchronize during most bursting events, which is also indicative of a weakly synchronized operational regime. Finally, simulated intraburst parameters of these "bimodal models" were also successfully verified to lie within the experimental ranges as described in Gritsun et al. (2010); this further supports the validity of these models.

As mentioned above, models with injected Gaussian noise (noise-driven simulations) usually resulted in IBIs with exponential distributions. However, most of the experimental groups showed IBI distributions with a unimodal peaked distribution, thereby reducing qualitatively the validity of the noise-driven model. To better match the IBI distribution of recorded data, we therefore replaced injected synaptic noise with deterministic synaptic input (pseudopacemaker-driven simulations). This yielded IBIs that no longer followed an exponential distribution. The new IBI distribution highly depended on the $F_{\mathrm{p}}$ distribution span $(\xi, \sigma)$ and location $(\mu)$ : a narrower span led to more regular IBIs (a narrower IBI distribution), and the high end of the $F_{\mathrm{p}}$ range produced very short IBIs. This approach holds the middle between the random noise-driven and intrinsic pacemakerdriven network models that we used to validate intraburst characteristics in a previous study (Gritsun et al. 2010). We adjusted the distribution of $F_{\mathrm{p}}$ to achieve maximum similarity between experimental and simulated IBI distributions. These models showed the best resemblance to the experimental interburst parameters, including the shape of the IBI distribution. Although there is some experimental data to support the concept of endogenously active cells (Latham et al. 2000), it is as yet unclear whether their firing is stochastic or deterministic. Our results suggest that their firing is deterministic rather than stochastic.

In a previous study we used network models with intrinsic pacemakers. In that approach, intrinsic spikes were evoked as the result of larger current influx into a neuron. Such simulations showed good agreement with experimental intraburst parameters (Gritsun et al. 2010). However, analysis of IBIs showed a poor resemblance to recorded data. The major drawback was that the networks produced periodic bursts only at several constant rates. Burst rates were effectively locked to the fastest pacemakers while other, slower pacemaker neurons were discharged by preceding bursts and could therefore not initiate bursts themselves. In contrast, in network models with pseudo-pacemakers the intense 
neurons received external pacemaker pulses. Now, they could fire again in spite of being discharged by a previous burst and thus initiate bursts themselves.

\section{Conclusions}

We found that experimental activity was always in the weakly synchronized regime. We showed that the results obtained from small network models cannot simply be extrapolated to models of more realistic size, and that larger networks with homogeneous synaptic strengths cannot operate in a weakly synchronized regime as commonly found in experimental recordings. Alternatively, noise-driven network models that include a set of intense neurons (with stronger connections to their targets) were able to produce IBIs in the experimental range. Addition of a set of intense neurons to the model yielded a robust weakly synchronized regime over a wide range of network parameter values and noise frequencies. Such models reproduced recorded data quite well but tended to overestimate the incidence of very short IBIs, as a direct consequence of the applied normally distributed input noise. Applying more deterministic input, with frequencies from a certain distribution (pseudo-pacemaker-driven models), reduced the incidence of very short IBIs and reproduced experimental data even better, but the plausibility of this approach remains to be confirmed. Models with intrinsic pacemakers showed rather poor performance as they failed to reproduce the wide ranges of experimentally observed IBIs. In this study we hypothesized a simple model of "first-to-fire" (so-called leader) neurons, which do not necessarily have a different sensitivity to noise, or stronger synaptic inputs, but require relatively strong synapses (by long-term potentiation) to their targets. Confirmation of this hypothesis requires more sophisticated modeling.

Acknowledgements The work presented in this article was funded by the EU Marie-Curie NEURoVERS-it project (MRTN-CT-2005019247).

Open Access This article is distributed under the terms of the Creative Commons Attribution Noncommercial License which permits any noncommercial use, distribution, and reproduction in any medium, provided the original author(s) and source are credited.

\section{References}

Amit DJ, Brunel N (1997) Dynamics of a recurrent network of spiking neurons before and following learning. Netw Comput Neural Syst 8:373-404

Baker RE, Corner MA, Pelt J (2006) Spontaneous neuronal discharge patterns in developing organotypic mega-co-cultures of neonatal rat cerebral cortex. Brain Res 1101:29-35

Bi GQ, Poo MM (1998) Synaptic modifications in cultured hippocampal neurons: dependence on spike timing, synaptic strength, and postsynaptic cell type. J Neurosci 18:10464-10472
Coles S (2001) An introduction to statistical modelling of extreme values. Springer-Verlag, London

Destexhe A, Badoual M, Piwkowska Z, Bal T, Rudolph M (2004) A novel method for characterizing synaptic noise in cortical neurons. Neurocomputing 58-60:191-196

Eckmann JP, Jacobi S, Marom S, Moses E, Zbinden C (2008) Leader neurons in population bursts of $2 \mathrm{D}$ living neural networks. New J Phys 10:015011

Eytan D, Marom S (2006) Dynamics and effective topology underlying synchronization in networks of cortical neurons. J Neurosci 26:8465-8476

French DA, Gruenstein EI (2006) An integrate-and-fire model for synchronized bursting in a network of cultured cortical neurons. J Comput Neurosci 21:227-241

Gibson JR, Connors BW (2003) Chemical and electrical synapses in neocortex. In: Arbib MA (ed) Handbook of brain theory and neural networks. 2 edn. MIT Press, Cambridge, pp $725-729$

Giugliano M, Darbon P, Arsiero M, Lüscher HR, Streit J (2004) Single-neuron discharge properties and network activity in dissociated cultures of neocortex. J Neurophysiol 92:977-996

Gritsun TA, le Feber J, Stegenga J, Rutten WLC (2010) Network bursts in cortical cultures are best simulated using pacemaker neurons and adaptive synapses. Biol Cybern 102:1-18

Hansel D, Sompolinsky H (1996) Chaos and synchrony in a model of a hypercolumn in visual cortex. J Comput Neurosci 3: $7-34$

Hubbard JI, Stenhouse D, Eccles RM (1967) Origin of synaptic noise. Science 157:330-331

Ichikawa M, Muramoto K, Kobayashi K, Kawahara M, Kuroda Y (1993) Formation and maturation of synapses in primary cultures of rat cerebral cortical cells: An electron microscopic study. Neurosci Res 16:95-103

Izhikevich EM (2003) Simple model of spiking neurons. IEEE Trans Neural Netw 14:1569-1572

Kitano K, Fukai T (2007) Variability v.s. synchronicity of neuronal activity in local cortical network models with different wiring topologies. J Comput Neurosci 23:237-250

Latham PE, Richmond BJ, Nirenberg S, Nelson PG (2000) Intrinsic dynamics in neuronal networks. II. Experiment. J Neurophysiol $83: 828-835$

le Feber J, Van Pelt J, Rutten WLC (2009) Latency-related development of functional connections in cultured cortical networks. Biophys J 96:3443-3450

le Feber J, Rutten WLC, Stegenga J, Wolters PS, Ramakers GJA, Pelt J (2007) Conditional firing probabilities in cultured neuronal networks: a stable underlying structure in widely varying spontaneous activity patterns. J Neural Eng 4:54-67

Maeda E, Robinson HPC, Kawana A (1995) The mechanisms of generation and propagation of synchronized bursting in developing networks of cortical neurons. J Neurosci 15:6834-6845

Mainen ZF, Sejnowski TJ (1995) Reliability of spike timing in neocortical neurons. Science 268:1503-1506

Markram H, Wang Y, Tsodyks M (1998) Differential signaling via the same axon of neocortical pyramidal neurons. Proc Natl Acad Sci USA 95:5323-5328

Muller TH, Swandulla D, Zeilhofer HU (1997) Synaptic connectivity in cultured hypothalamic neuronal networks. J Neurophysiol 77:3218-3225

Nesse WH, Borisyuk A, Bressloff PC (2008) Fluctuation-driven rhythmogenesis in an excitatory neuronal network with slow adaptation. J Comput Neurosci 25:317-333

Rodriguez-Molina VM, Aertsen A, Heck DH (2007) Spike timing and reliability in cortical pyramidal neurons: effects of EPSC kinetics, input synchronization and background noise on spike timing. PLoS One 2:e319 
Romijn HJ, Van Huizen F, Wolters PS (1984) Towards an improved serum-free, chemically defined medium for long-term culturing of cerebral cortex tissue. Neurosci Biobehav Rev 8:301-334

Scott DW (1979) On optimal and data-based histograms. Biometrika 66:605-610

Segev R, Shapira Y, Benveniste M, Ben-Jacob E (2001) Observations and modeling of synchronized bursting in two-dimensional neural networks. Phys Rev 64:011920

Segev R, Benveniste M, Hulata E, Cohen N, Palevski A, Kapon E, Shapira Y, Ben-Jacob E (2002) Long term behavior of lithographically prepared in vitro neuronal networks. Phys Rev Lett 88:1181021-1181024

Song S, Miller KD, Abbott LF (2000) Competitive Hebbian learning through spike-timing-dependent synaptic plasticity. Nat Neurosci 3:919-926

Stegenga J, le Feber J, Marani E, Rutten WLC (2008) Analysis of cultured neuronal networks using intra-burst firing characteristics. IEEE Trans Biomed Eng 55(4):1382-1390

Stevens CF, Zador AM (1998) Input synchrony and the irregular firing of cortical neurons. Nat Neurosci 1:210-217

Strata F, Atzori M, Molnar M, Ugolini G, Tempia F, Cherubini E (1997) A pacemaker current in dye-coupled hilar interneurons contributes to the generation of giant GABAergic potentials in developing hippocampus. J Neurosci 17:1435-1446

Teich MC, Saleh BEA, Perina J (1984) Role of primary excitation statistics in the generation of antibunched and sub-poisson light. J Opt Soc Am B 1:366-389
Toledo-Rodriguez M, Gupta A, Wang Y, Wu CZ, Markram H (2003) Neocortex: basic neuron types. In: Arbib MA (ed) Handbook of brain theory and neural networks. 2 edn. MIT Press, Cambridge, pp 719-725

Tsodyks M, Pawelzik K, Markram H (1998) Neural networks with dynamic synapses. Neural Comput 10:821-835

Tsodyks M, Uziel A, Markram H (2000) Synchrony generation in recurrent networks with frequency-dependent synapses. J Neurosci 20:RC50

Van Pelt J, Wolters PS, Corner MA, Rutten WLC, Ramakers GJA (2004) Long-term characterization of firing dynamics of spontaneous bursts in cultured neural networks. IEEE Trans Biomed Eng 51:2051-2062

Vladimirski BB, Tabak J, O’Donovan MJ, Rinzel J (2008) Episodic activity in a heterogeneous excitatory network, from spiking neurons to mean field. J Comput Neurosci 25:39-63

Wagenaar DA, Pine J, Potter SM (2006) An extremely rich repertoire of bursting patterns during the development of cortical cultures. BMC Neurosci 7:11

Wiedemann UA, Luthi A (2003) Timing of network synchronization by refractory mechanisms. J Neurophysiol 90:3902-3911

Zhang LI, Tao HW, Holt CE, Harris WA, Poo MM (1998) A critical window for cooperation and competition among developing retinotectal synapses. Nature 395:37-44 